

University of Central Florida

STARS

$1-1-2014$

\title{
Two-dimensional fermionic Hong-Ou-Mandel interference with massless Dirac fermions
}

\author{
M. A. Khan \\ University of Central Florida \\ Michael N. Leuenberger \\ University of Central Florida
}

Find similar works at: https://stars.library.ucf.edu/facultybib2010 University of Central Florida Libraries http://library.ucf.edu

This Article is brought to you for free and open access by the Faculty Bibliography at STARS. It has been accepted for inclusion in Faculty Bibliography 2010 s by an authorized administrator of STARS. For more information, please contact STARS@ucf.edu.

\section{Recommended Citation}

Khan, M. A. and Leuenberger, Michael N., "Two-dimensional fermionic Hong-Ou-Mandel interference with massless Dirac fermions" (2014). Faculty Bibliography 2010s. 5567.

https://stars.library.ucf.edu/facultybib2010/5567

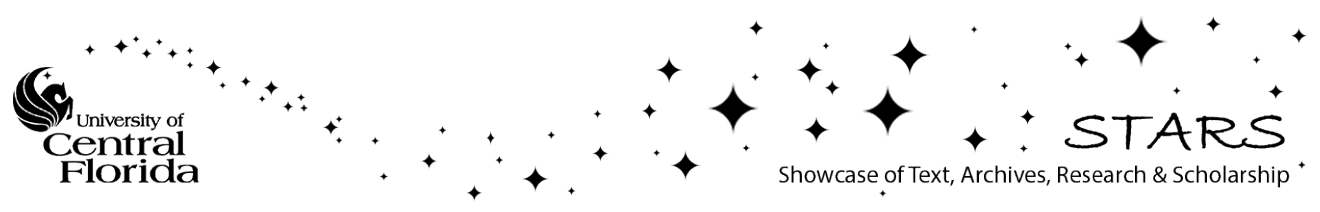




\title{
Two-dimensional fermionic Hong-Ou-Mandel interference with massless Dirac fermions
}

\author{
M. A. Khan ${ }^{1,2,3}$ and Michael N. Leuenberger ${ }^{1,2, *}$ \\ ${ }^{1}$ NanoScience Technology Center, University of Central Florida, Orlando, Florida 32826, USA \\ ${ }^{2}$ Department of Physics, University of Central Florida, Orlando, Florida 32816, USA \\ ${ }^{3}$ Federal Urdu University of Arts, Science and Technology, Islamabad, Pakistan
}

(Received 23 May 2014; revised manuscript received 7 August 2014; published 29 August 2014)

\begin{abstract}
We propose a two-dimensional Hong-Ou-Mandel (HOM) type interference experiment for massless Dirac fermions in graphene and 3D topological insulators. Since massless Dirac fermions exhibit linear dispersion, similar to photons in vacuum, they can be used to obtain the HOM interference intensity pattern as a function of the delay time between two massless Dirac fermions. We show that while the Coulomb interaction leads to a significant change in the angle dependence of the tunneling of two identical massless Dirac fermions incident from opposite sides of a potential barrier, it does not affect the HOM interference pattern. We apply our formalism to develop a massless Dirac fermion beam splitter (BS) for controlling the transmission and reflection coefficients. We calculate the resulting time-resolved correlation function for two identical massless Dirac fermions scattering off the BS.
\end{abstract}

DOI: 10.1103/PhysRevB.90.075439

PACS number(s): 85.35.Ds, 72.80.Vp, 04.50.-h

\section{INTRODUCTION}

When two indistinguishable bosons are incident on opposite sides of a 50/50 beam splitter (BS), Bose-Einstein quantum statistics demands bunching, i.e., the outgoing bosons must leave together in one of the two outputs, which was first observed with photons in the Hong-Ou-Mandel (HOM) experiment [1]. Observation of zero coincidence for simultaneous photons is identified by a dip in the correlation function and rises with time delay [1]. HOM-type interference has been utilized in quantum tests of non locality [2] and can be used to investigate the degree of indistinguishability of the incident particles. Also, the HOM experiment is one of the key elements of linear-optics-based quantum computation [3]. Several experiments have already demonstrated the HOM interference with photons [1,4], plasmons [5], levitons [6], and electrons [7-9]. Interestingly, it is possible to replace the bosons in the HOM interference experiment by fermions, which leads to the exact opposite behavior. Due to the FermiDirac quantum statistics, fermions appear in different outputs, as identical fermions have the tendency of antibunching over small distances, leading to a peak in the coincidence measurement at zero delay. While photons in vacuum exhibit a linear dispersion relation, electrons in gapped semiconductor materials typically have a quadratic dispersion relation, which is a major obstacle for observing the fermionic analog of the HOM interference due to the spreading of electronic wave function. In order to overcome this obstacle, it is essential to identify physical systems where the electrons have a linear dispersion relation.

One such example is the one-dimensional edge states of quantum Hall systems exhibiting ballistic conductance and linear dispersion, where the one-dimensional fermionic HOM experiment [9] has been successfully implemented. Similar results are expected theoretically for quantum spin Hall states [10]. In order to create a two-dimensional fermionic

*michael.leuenberger@ucf.edu
HOM interference pattern, we need fermionic particles with a linear dispersion relation in two dimensions. Ideal candidates are massless Dirac fermions in graphene [11] and on the surface of three-dimensional (3D) topological insulators [12]. Here we show that it is possible to create a two-dimensional fermionic HOM interference pattern by considering the scattering of two massless Dirac fermions in the case of a rectangular potential barrier. We show that at specific incident angles a 50/50 BS for massless Dirac fermions can be realized, even when considering the Coulomb interaction between the massless Dirac fermions. Interestingly, the Coulomb interaction leads to a substantial change in angle distribution of the transmission and reflection coefficients. The idea of using a potential barrier as a beam splitter has already been proposed in the context of a two-dimensional electron gas in a wide-band-gap semiconductor [13]. In Ref. [9] electronic HOM interference is demonstrated by using quantum Hall edge states. This experiment shows that the cross-correlation function at zero time delay is reduced, which has been attributed to the reduction in indistinguishability due to the interaction between the copropagating edge states [14]. Here, in the case of massless Dirac fermions in graphene and 3D topological insulators, we show that within the eikonal approximation the Coulomb interaction between the two incident massless Dirac fermions at the beam splitter does not affect the correlation function, which thus is solely determined by the quantum statistics of the particles.

In the case of one-dimensional edge states in topological insulators, the antibunching of two counterpropagating electrons with opposite spins is known to be due to time reversal symmetry, commonly referred to as $Z_{2}$ dip [10]. The $Z_{2}$ dip in the noise comes from the fact that there is no backscattering from any Hermitian potential $V$ in a topological insulator, including a Hermitian beam splitter. Our results are in agreement with the $Z_{2}$ dip for normal incidence. However, no such phenomenon is found for the massless Dirac fermions in graphene because the real spin is not locked to the momentum due to the negligibly small spin-orbit coupling in graphene. 
The realization of fermionic HOM interference experiment is provided by a three-step process: (i) generation of a single electron source, (ii) construction of the BS, which is the primary focus of this work, and (iii) detection of and counting the coincidences. In Ref. [15] a single electron pump (SEP) in graphene has been demonstrated experimentally. The pump is made of two lithographically defined graphene islands that are coupled to each other and to source and drain contacts by narrow constrictions. By rapid modulation of the gate voltages, a single electron can be pumped to the drain contact. The frequency $f$ of the oscillating voltage applied to the gates determines the rate at which a single charge is transferred and thus the size of the pump current $I=e f$. The frequency $f$ is of the order of GHz. As stated by the authors in Ref. [15], this single electron pump can be used as a single massless Dirac fermion source in graphene in the field of electron quantum optics. This setup is not constrained to edge states, but can also be used for a two-dimensional graphene sheet. The basic idea of this setup can be transferred to the case of massless electrons in topological insulators. A scanning probe microscope (SPM) tip can be used to image quantum interference and raylike propagation of electrons [16]. Alternatively, the interference can be probed by measuring the fluctuations of electrical current in the outputs channels related to the fluctuations of number of particles transmitted [9].

The manuscript is organized as follows. In Sec. II we define the model Hamiltonians for graphene and 3D topological insulators. In Sec. III we calculate the phase change due to the Coulomb interaction within the eikonal approximation. Section IV describes the tunneling through a potential barrier, including the Coulomb interaction due to scattering of two massless Dirac fermions. The evaluation of the twodimensional fermionic HOM interference is performed in Sec. V.

\section{MODEL}

It was shown [17] that the transmission probability $T$ of massless Dirac fermions (in graphene) with energy $E$ through a rectangular potential barrier of height $V_{0}$ and width $D$ varies as a function of incident angle $\phi .100 \%$ transmission probability is observed at normal incidence $\phi=0$, a feature known as Klein tunneling. Exactly the same result can be obtained for surface electronic states of 3D topological insulators. The reason for this coincidence is that in both systems the dynamics of electrons is defined by similar Hamiltonians. The only difference between the two systems is that in graphene the pseudospin is locked parallel to the linear momentum and in 3D topological insulators the real spin is locked perpendicular to linear momentum, respectively, i.e.,

$$
\hat{H}_{0, g}=v_{F} \boldsymbol{\sigma} \cdot \mathbf{p}, \quad \hat{H}_{0, T I}=v_{\mathrm{eff}}(\boldsymbol{\sigma} \times \mathbf{p})_{z},
$$

where $\sigma_{i}$ 's are Pauli matrices, corresponding to the pseudospin in the case of graphene and to the real spin in the case of 3D topological insulators, respectively, and $\mathbf{p}$ is the momentum operator. The angle-dependent transmission probability through a potential barrier can be used to make a BS for massless Dirac fermions. For observing the HOM-type interference we need to inject two massless Dirac fermions from the opposite sides

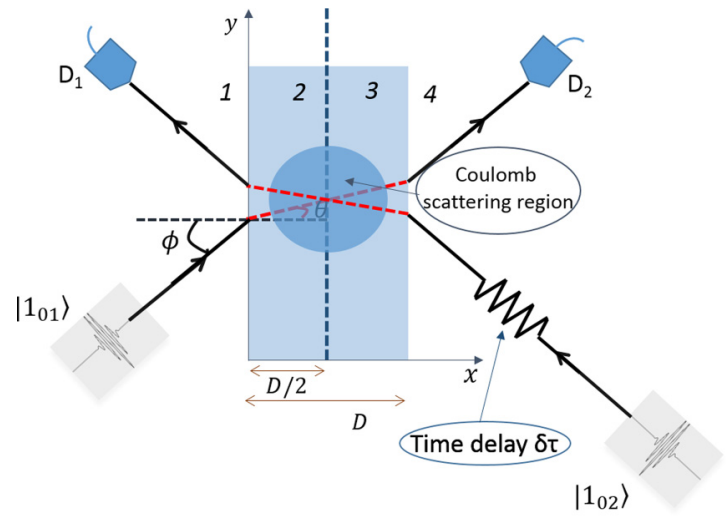

FIG. 1. (Color online) HOM experiment with massless Dirac fermion BS.

of the barrier, as shown in Fig. 1, and their transmissions and reflections will produce the desired interference.

\section{COULOMB INTERACTION IN THE EIKONAL APPROXIMATION}

We take advantage of the eikonal approximation [18] to calculate the phase change acquired by a massless Dirac electron when scattering from a second massless Dirac electron due to the Coulomb interaction. First it is important to verify the validity of the eikonal approximation. Inside the barrier kinetic energy of the massless Dirac fermions is described by $\left|V_{0}-E\right|$, where $V_{0}$ is the height of the barrier and $E$ is the energy of the incident particles. Here we consider the screened Coulomb potential, represented by the Yukawa potential $V(r)=U_{0} e^{-\mu r) / \mu r}$, where $\mu^{-1}$ is the screening length. For graphene $\mu=g_{s} g_{v} e^{2} k_{F} / 4 \pi \kappa \epsilon_{0} \hbar v_{F}, \kappa$ is the background lattice dielectric constant, $U_{0}=e^{2} \mu / 4 \pi \kappa \epsilon_{0}$, and $k_{F}$ is the Fermi wave vector. $g_{v}$ and $g_{s}$ are valley and spin degeneracies, respectively. By averaging the Yukawa potential over five screening lengths we obtain

$$
\langle V(r)\rangle=\frac{1}{\int_{0}^{5 / \mu} d^{2} r} \int_{0}^{5 / \mu} V(r) d^{2} r \approx 10 \mathrm{meV} .
$$

After five screening lengths the strength of the Yukawa potential can be approximated to be zero. We take the values of $E=80 \mathrm{meV}$ and $V_{0}=200 \mathrm{meV}$ so that $\langle V(r)\rangle \ll\left|V_{0}-E\right|$.

Although we solve the Coulomb scattering for massless Dirac fermions in graphene, our results are general and applicable to surface states of 3D topological insulators as well because the Coulomb interaction does not depend on the spin. Working in the eikonal approximation, the exact wave function $\Psi$ of the Hamiltonian $H=H_{0}+V(r)$ can be approximated by

$$
\Psi \sim\left(\begin{array}{l}
\alpha \\
\beta
\end{array}\right) e^{i S(r) / \hbar} .
$$

Starting from the Dirac equation shown in Eq. (1) and expanding in powers of $\hbar$, we obtain in zeroth order the relativistic Hamilton-Jacobi equation

$$
\left|\partial_{x} S(r)\right|^{2}+\left|\partial_{y} S(r)\right|^{2} \approx E^{2} / v_{F}^{2}-2 V(r) E / v_{F}^{2} .
$$


We compute $S(r)$ from Eq. (4) by assuming that the trajectory is a straight line, which is valid for large energies and small deflection angles [18]. Equation (4) then yields in linear approximation in $V$,

$$
\frac{S(x)}{\hbar} \approx k x-\frac{1}{\hbar v_{F}} \int_{-\infty}^{x} 2 V\left(b^{\prime}, x^{\prime}\right) d x^{\prime},
$$

where $b^{\prime}$ is the impact parameter [18]. Similar to the nonrelativistic derivation [18], we obtain the relativistic scattering amplitude

$$
f\left(\mathbf{k}, \mathbf{k}^{\prime}\right)=-i \sqrt{\frac{k}{2 \pi}} \int_{-\infty}^{\infty} d b e^{-i k b \theta}\left[e^{2 i \xi\left(b^{\prime}\right)}-1\right],
$$

where $\xi\left(b^{\prime}\right)=-\frac{1}{2 \hbar v_{F}} \int_{-\infty}^{\infty} d x^{\prime} V\left(b^{\prime}, x^{\prime}\right)$ and $\theta$ is the angle between $\mathbf{k}$ and $\mathbf{k}^{\prime}$. Equation (6) is in agreement with the optical theorem in scattering theory [18]. Equation (6) can be solved for the screened Coulomb potential, i.e., the Yukawa potential with $V\left(b^{\prime}, x^{\prime}\right)=U_{0} \exp \left(-\mu \sqrt{b^{\prime 2}+x^{\prime 2}}\right) / \mu \sqrt{b^{\prime 2}+x^{\prime 2}}$. In the laboratory frame $\theta \longrightarrow \theta / 2$. The phase change $\Delta$ in the forward direction acquired by the particle while passing through the scattering region can be evaluated by setting $|\mathbf{k}|=\left|\mathbf{k}^{\prime}\right|=k_{F}$ for elastic scattering, i.e.,

$$
\Delta=\lim _{\theta \rightarrow 0} \operatorname{Re}\left(\sqrt{k_{F}} f\left(\mathbf{k}, \mathbf{k}^{\prime}\right)\right)=-\frac{\sqrt{2 \pi} U_{0}}{\hbar v_{F} \mu} \frac{k_{F}}{\mu} .
$$

\section{TUNNELING THROUGH RECTANGULAR POTENTIAL}

It is now straightforward to solve the tunneling problem shown in Fig. 1. The electron is incident on the barrier from the right at an angle $\phi$ with respect to the $x$ axis. It propagates at an angle $\theta$ in region 2 and is transmitted in region 3 at the same angle $\phi$. Following Ref. [17], the components of the massless Dirac spinor $\Psi_{1}$ and $\Psi_{2}$ can be written as $\Psi_{i}(x, y)=$ $\Psi_{i}(x) e^{i k_{y} y}, i=1,2$, with

$$
\begin{gathered}
\Psi_{1}(x)=\left\{\begin{array}{ll}
e^{i k_{x} x}+r e^{-i k_{x} x} & x<0 \\
a e^{i q_{x} x}+b e^{-i q_{x} x} & 0<x<\frac{D}{2} \\
a e^{i q_{x} x+i \Delta}+b e^{-i q_{x} x-i \Delta} & \frac{D}{2}<x<D \\
t^{\prime} e^{i k_{x} x+i \Delta} & x>D
\end{array},\right. \\
\Psi_{2}(x)=\left\{\begin{array}{ll}
s\left[e^{i k_{x} x+i \phi}-r e^{-i k_{x} x-i \phi}\right] & x<0 \\
s^{\prime}\left[a e^{i q_{x} x+i \theta}-b e^{-i q_{x} x-i \theta}\right] & 0<x<\frac{D}{2} \\
s^{\prime}\left[a e^{i q_{x} x+i \theta+i \Delta}-b e^{-i q_{x} x-i \theta-i \Delta}\right] & \frac{D}{2}<x<D \\
s t^{\prime} e^{i k_{x} x+i \phi+i \Delta} & x>D
\end{array},\right.
\end{gathered}
$$

where $k_{x}=k_{F} \cos \phi, k_{y}=k_{F} \sin \phi$ are the components of the wave vector outside the barrier and $q_{x}=$ $\sqrt{\left(E-V_{0}\right)^{2} /\left(\hbar v_{F}\right)^{2}-k_{y}^{2}}$ and $\tan \theta=k_{y} / q_{x}$ is the refraction angle, $s=\operatorname{sgn}(E)$ and $s^{\prime}=\operatorname{sgn}\left(E-V_{0}\right)$. The transmission coefficient $t^{\prime}$ can be evaluated by using the continuity conditions at $x=0$ and $x=D$ and is

$$
\begin{aligned}
t^{\prime}= & 2 \exp \left(-i k_{x} D\right) \cos \theta \cos \phi /\left\{s s ^ { \prime } \left[e^{-i\left(q_{x} D+\Delta\right)} \cos (\theta+\phi)\right.\right. \\
& \left.\left.+e^{i\left(q_{x} D+\Delta\right)} \cos (\theta-\phi)\right]-2 i \sin \left(q_{x} D+\Delta\right)\right\}
\end{aligned}
$$

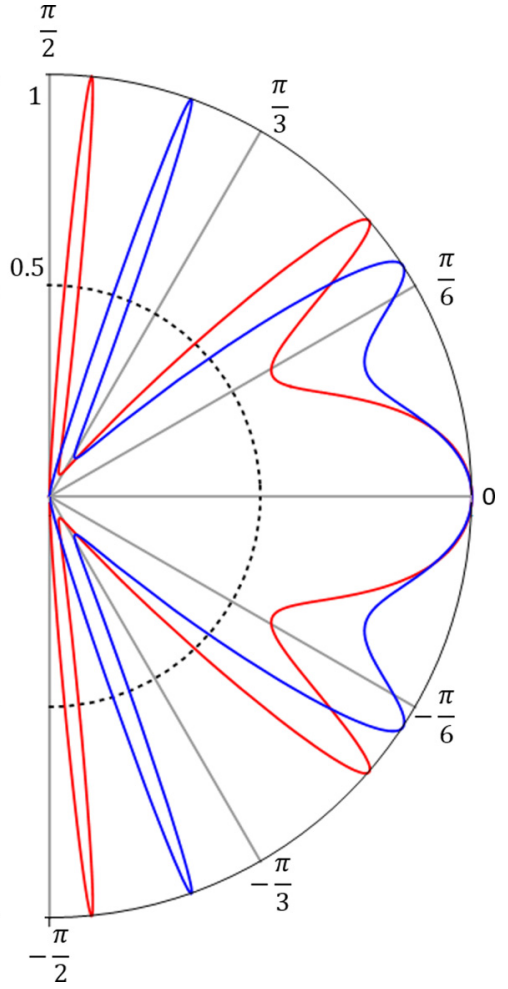

FIG. 2. (Color online) Transmission probability $T$ as a function of incident angle $\phi$. The electron concentration $n$ outside the barrier is chosen as $0.5 \times 10^{12} \mathrm{~cm}^{-2}$. This corresponds to a Fermi energy and wavelength of incident electrons of $E_{F} \approx 80 \mathrm{meV}$ and $\lambda \approx 50$ $\mathrm{nm}$, respectively. The barrier height $V_{0}=200 \mathrm{meV}$. The red curve is the solution for $\Delta=0$ and the blue curve is the solution for $\Delta=-0.63$. Black (dashed) semicircle is drawn at $50 \%$ transmission probability.

In Fig. 2 the transmission coefficient $T=t^{* *} t^{\prime}$ is plotted as a function of incident angle $\phi$ for the cases when $\Delta=0$ (red curve) and $\Delta=-\sqrt{2 \pi} U_{0} k_{F} / \hbar v_{F} \mu^{2}$ (blue curve). Interestingly, the Coulomb interaction results in a substantial shift of the transmission peaks while preserving Klein tunneling. This result is in agreement with previous findings [19]. It has been experimentally demonstrated that Coulomb interaction results in the renormalization of the Fermi velocity in graphenelike systems and fails to create any energy gap. The energy gap is the only possibility for the removal of Klein tunneling [19]. In the limit $V_{0} \ll E$,

$$
T=\frac{\cos ^{2} \phi}{1-\cos ^{2}\left(q_{x} D+\Delta\right) \sin ^{2} \phi} .
$$

For normal incidence $T=1$, regardless of the height and width of the barrier. Away from normal incidence, the other transmission peaks correspond to the condition of constructive interference, which occurs when $q_{x} D+\Delta=n \pi$, where $n=$ $0, \pm 1, \pm 2, \ldots$. Comparing Eq. (11) with the result in Ref. [17] there is an additional phase $\Delta$ in the denominator, which comes from the Coulomb interaction. It can be seen from Fig. 2 that at certain angles $T=50 \%$. For these angles of incidence, this 
modified barrier can be used as a 50/50 BS. At the same $\phi$, the Coulomb interaction leads to an asymmetry in $T$ and $R$. In addition, we can change $T$ and $R$ to any desired value ranging between 0 and 1 by tuning $\phi$.

\section{FERMIONIC HONG-OU-MANDEL INTERFERENCE}

The schematic diagram of the HOM experiment is shown in Fig. 1. It consists of two wave packets triggered by two SEPs, a BS (orange line) and two SPM tips (blue pentagons) to measure the electron flux [16]. The BS is considered to be lossless, i.e., $T+R=1$. Let us now consider two massless Dirac fermions that are incident on the BS from opposite sides. Let $\tau_{1}$ be the time it takes for the electrons to get from the source to the detector. We define $\delta \tau$ as the time delay between the two incident electrons. $\delta \tau$ can be introduced either by displacing the position of the BS towards one of the sources or by introducing the time delay between the switching pulses of the two SEPs. Our goal is to calculate the correlation function corresponding to the coincidence counts at the two detectors as a function of the time delay $\delta \tau$. The inputs of the BS are described by the indices 01,02 , i.e., $c_{01}^{\dagger}\left|0_{01}, 0_{02}\right\rangle=\left|1_{01}, 0_{02}\right\rangle$ and $c_{02}^{\dagger}\left|0_{01}, 0_{02}\right\rangle=\left|0_{01}, 1_{02}\right\rangle$, where $c_{01}^{\dagger}\left(c_{01}\right)$ are electron creation (annihilation) operators. We omit the spin index because we assume that the two electrons have parallel spins. The $Z_{2}$ dip is associated with the massless Dirac fermions of opposite spin for normal incidence in topological insulators, as mentioned earlier. As our main focus here is to investigate the HOM-type interference, a consequence of the indistinguishability of quantum particles, electrons of the same spin are considered. Similarly, the outputs are described by the indices 1,2 . The output operators are related to the input operators through the following linear scattering relations:

$$
\begin{aligned}
& \hat{c}_{1}(t)=\sqrt{T} \hat{c}_{01}\left(t-\tau_{1}\right)+i \sqrt{R} \hat{c}_{02}\left(t-\tau_{1}+\delta \tau\right), \\
& \hat{c}_{2}(t)=\sqrt{T} \hat{c}_{02}\left(t-\tau_{1}\right)+i \sqrt{R} \hat{c}_{01}\left(t-\tau_{1}-\delta \tau\right),
\end{aligned}
$$

where $i$ corresponds to a $\pi / 2$ phase shift and $\hat{c}_{0 j}(t)=$ $\xi_{j}(t) \hat{c}_{0 j} . \xi_{j}(t)$ is the distribution function in time, considering a general temporal form of the wave packet $\xi_{j}(t)=$ $\Theta(t) \exp \left(-\Gamma_{j} t / 2\right) \exp (i \omega t)$ [9]. $\Theta(t)$ is the Heaviside step function and $\Gamma_{j}$ is the SEP emission rate of the electron. The correlation function describing the joint probability of detection of electrons at the two detectors at times $t$ and $t+\tau$ is

$$
P_{12}(t)=C\left\langle 0\left|\hat{c}_{02} \hat{c}_{01} \hat{c}_{1}^{\dagger}(t) \hat{c}_{2}^{\dagger}(t+\tau) \hat{c}_{2}(t+\tau) \hat{c}_{1}(t) \hat{c}_{01}^{\dagger} \hat{c}_{02}^{\dagger}\right| 0\right\rangle .
$$

$C$ is the normalization constant. This can readily be evaluated by means of Eqs. (12) and (13). The number of coincidence counts $N_{c}(1,2)$ can be obtained by integrating $P_{12}(t)$ over time $t$. This yields

$$
\begin{aligned}
\frac{N_{c}(\delta \tau)}{C} \Gamma_{1} \Gamma_{2}= & \tilde{N}_{c}(\delta \tau)=T^{2}+R^{2}+R T \frac{8 \Gamma_{1} \Gamma_{2}}{\left(\Gamma_{1}+\Gamma_{2}\right)^{2}} \\
& \times\left\{\exp \left(\Gamma_{1} \delta \tau\right) \Theta(-\delta \tau)+\exp \left(-\Gamma_{2} \delta \tau\right) \Theta(\delta \tau)\right\},
\end{aligned}
$$

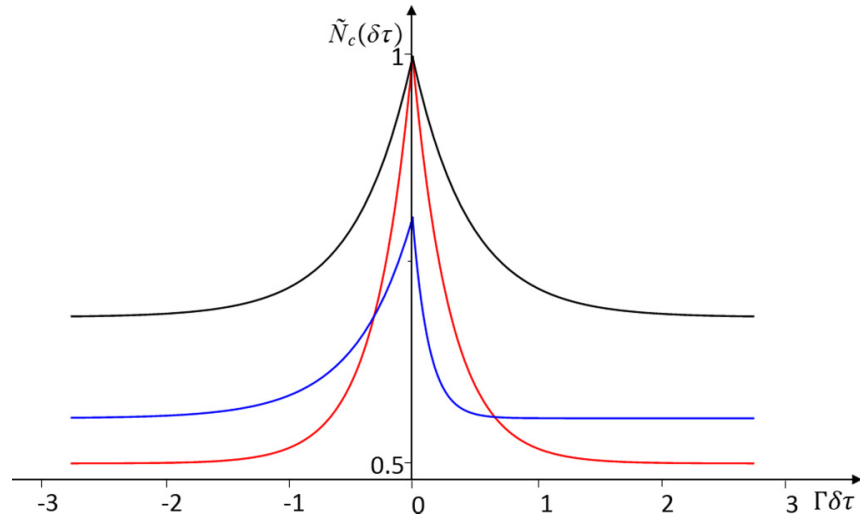

FIG. 3. (Color online) Interference peak for normalized number of coincidences $\widetilde{N}_{c}(\delta \tau)$ against time delay $\delta \tau$. The red curve is for $R=T=1 / 2$ and for $\Gamma_{1}=\Gamma_{2}=\Gamma$, and the black curve is for $R=$ $1 / 5, T=4 / 5$ and for $\Gamma_{1}=\Gamma_{2}=2 \Gamma / 3$. The blue curve is for $T=$ $2 / 3, R=1 / 3$ and $\Gamma_{1}=2 \Gamma / 3, \Gamma_{2}=7 \Gamma / 3$, where $\Gamma=3 \times 10^{-12} \mathrm{~s}^{-1}$.

where $\widetilde{N}_{c}(\delta \tau)$ is the normalized number of coincidences. Equation (15) is our main result. The coincidence counts depend both on the time delay $\delta \tau$ and the transmission and reflection coefficients. The coincidence counts can be tuned by introducing an asymmetry in the reflection and transmission coefficients. For perfect transmissions and reflections $\widetilde{N}_{c}(\delta \tau)$ remains at unity regardless of the value of $\delta \tau$. For large $\delta \tau$ the third term on the right-hand side of Eq. (15) goes to zero, and the expression for the coincidence counts reduces to $T^{2}+R^{2}$. In case of identical electron sources, i.e., $\Gamma_{1}=\Gamma_{2}$, Eq. (15) simplifies to

$$
\begin{aligned}
\tilde{N}_{c}(\delta \tau)= & T^{2}+R^{2}+2 R T\left\{\exp \left(\Gamma_{1} \delta \tau\right) \Theta(-\delta \tau)\right. \\
& \left.+\exp \left(-\Gamma_{2} \delta \tau\right) \Theta(\delta \tau)\right\} .
\end{aligned}
$$

Note that for $\delta \tau=0 \widetilde{N}_{c}(\delta \tau)=(T+R)^{2}=1$, no matter what the values of $T$ and $R$ are, which reflects the antibunching of fermions. In Fig. 3 we plot the coincidence counts for different values of $R, T$, and $\Gamma$ (blue) as a function of the time delay $\delta \tau$. For the asymmetric case, i.e., for incident electrons with different decay rates, the HOM peak is substantially reduced, as can be seen in Fig. 3. This decrease in the coincidence counts is accounted for by the reduction in degree of indistinguishability of incident wave packets and is in agreement with existing literature [20].

If we consider the effect of the bandwidth of the wave packet in the case of the Coulomb interaction, it is possible for the Coulomb interaction to induce decoherence. However, in our case the Fermi energy is much larger than the bandwidth, i.e., $E_{F} \approx 80 \mathrm{meV} \gg \Gamma \approx 1 \mathrm{meV}$, giving rise to about $1 \%$ spread in the phase $\Delta$ in Eq. (7). Therefore the interaction-induced decoherence is negligibly small.

\section{CONCLUSION}

We developed the theoretical model of the two-dimensional HOM-type interference with massless Dirac fermions in graphene and in 3D topological insulators. The twodimensional setup allows for the tuning of the transmission 
and reflection coefficients by varying the angle of incidence of the two massless Dirac fermions. We provide the description of a realistic BS for massless Dirac fermions, including the effects of Coulomb interaction. Our results show that the Coulomb interaction does not affect the fermionic HOM peak (Pauli peak) for massless Dirac fermions within the eikonal approximation. However, asymmetry in the emission rates of the two massless Dirac fermions lifts the indistinguishability and this leads to the reduction of the HOM peak. The larger the asymmetry, the smaller is the peak.

\section{ACKNOWLEDGMENTS}

We acknowledge support from the NSF (Grants No. ECCS0901784 and No. ECCS-1128597) and the AFOSR (Grant No. FA9550-09-1-0450).
[1] C. K. Hong, Z. Y. Ou, and L. Mandel, Phys. Rev. Lett. 59, 2044 (1987).

[2] J. Torgerson, D. Branning, C. Monken, and L. Mandel, Phys. Lett. A 204, 323 (1995).

[3] E. Knill, R. Laflamme, and G. J. Milburn, Nature (London) 409, 46 (2001).

[4] J. Beugnon, M. P. A. Jones, J. Dingjan, B. Darquié, G. Messin, A. Browaeys, and P. Grangier, Nature (London) 440, 779 (2006).

[5] R. W. Heeres, L. P. Kouwenhoven, and V. Zwiller, Nat. Nanotechnol. 8, 719 (2013).

[6] J. Dubois, T. Jullien, F. Portier, P. Roche, A. Cavanna, Y. Jin, W. Wegscheider, P. Roulleau, and D. C. Glattli, Nature (London) 502, 659 (2013).

[7] J. R. Petta, H. Lu, and A. C. Gossard, Science 327, 669 (2010).

[8] G. Burkard, Science 327, 650 (2010); G. Burkard, D. Loss, and E. V. Sukhorukov, Phys. Rev. B 61, 16303 (2000).

[9] E. Bocquillon, V. Freulon, J.-M. Berroir, P. Degiovanni, B. Plaçais, A. Cavanna, Y. Jin, and G. Fève, Science 339, 1054 (2013); S. Ol' khovskaya, J. Splettstoesser, M. Moskalets, and M. Büttiker, Phys. Rev. Lett. 101, 166802 (2008).

[10] D. Ferraro, C. Wahl, J. Rech, T. Jonckheere, and T. Martin, Phys. Rev. B 89, 075407 (2014); A. Inhofer and D. Bercioux, ibid. 88, 235412 (2013); P. P. Hofer and M. Büttiker, ibid. 88, 241308(R) (2013); J. M. Edge, J. Li, P. Delplace, and M. Büttiker, Phys. Rev. Lett. 110, 246601 (2013).
[11] A. K. Geim and K. S. Novoselov, Nat. Mater. 6, 183 (2007); A. H. Castro-Neto, F. Guinea, N. M. R. Peres, K. S. Novoselov, and A. K. Geim, Rev. Mod. Phys. 81, 109 (2009).

[12] H. Zhang, C.-X. Liu, X.-L. Qi, X. Dai, Z. Fang, and S.-C. Zhang, Nat. Phys. 5, 438 (2009).

[13] C. W. J. Beenakker, C. Emary, M. Kindermann, and J. L. van Velsen, Phys. Rev. Lett. 91, 147901 (2003).

[14] C. Wahl, J. Rech, T. Jonckheere, and T. Martin, Phys. Rev. Lett. 112, 046802 (2014).

[15] M. R. Connolly, K. L. Chiu, S. P. Giblin, M. Kataoka, J. D. Fletcher, C. Chua, J. P. Grifths, G. A. C. Jones, V. I. Falko, C. G. Smith, and T. J. B. M. Janssen, Nat. Nanotechnol. 8, 417 (2013).

[16] M. A. Topinka, B. J. LeRoy, S. E. J. Shaw, E. J. Heller, R. M. Westervelt, K. D. Maranowski, and A. C. Gossard, Science 289, 2323 (2000); M. A. Topinka, B. J. LeRoy, R. M. Westervelt, S. E. J. Shaw, R. Fleischmann, E. J. Heller, K. D. Maranowski, and A. C. Gossard, Nature (London) 410, 183 (2001).

[17] M. I. Katsnelson, K. S. Novoselov, and A. K. Geim, Nat. Phys. 2, 620 (2006).

[18] J. J. Sakurai, Modern Quantum Mechanics (Addison Wesley, Reading, MA, 1994).

[19] V. N. Kotov, B. Uchoa, V. M. Pierre, F. Guinea, A. H. Castero Neto, Rev. Mod. Phys. 84, 1067 (2012).

[20] T. Jonckheere, J. Rech, C. Wahl, and T. Martin, Phys. Rev. B 86, 125425 (2012). 